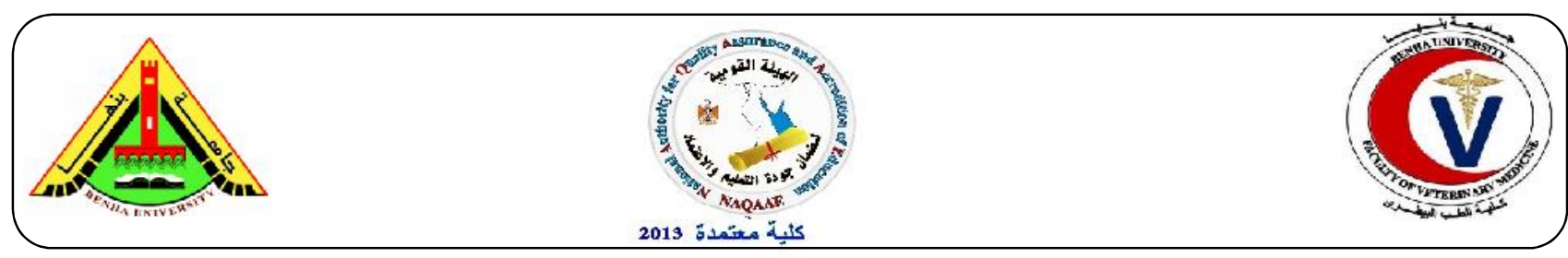

\title{
Immunohistochemical studies on thymus gland of rabbits after vaccination with viral hemorrhagic septicemia vaccine
}

\author{
Reda, I.E ${ }^{1}$, AboSalem, M.E. ${ }^{2}$, El Zoghby, E.M. ${ }^{1}$, Attia, H.F. ${ }^{1}$ and Emam, M.A. ${ }^{1}$ \\ ${ }^{1}$ Department of Histology, Faculty of Veterinary Medicine, Benha University \\ ${ }^{2}$ Department of Forensic Medicine and Toxicology, Faculty of Veterinary Medicine, Benha University
}

\section{A B S T R A C T}

The present study was carried out to investigate the structure of thymus of rabbits and cellular immune response after vaccination with viral haemorhagic septicemia vaccine. The present work was carried out on fifteen apparently healthy rabbits which were divided into two groups, control non-vaccinated and vaccinated. The rabbits were humanly sacrificed, eviscerated and subsequently tissue specimens from the thymus were taken for histological and immunohistochemical studies of thymus. Serum samples were collected for measurement of cytokines, interleukin 2 and 6 (IL-2 and IL-6) and tumer necrosis factor alpha (TNF $\alpha$ ). Present results showed that the thymus was lobulated organ consisted of peripheral dark cortex and central light stained medulla. Cortex consisted of high population of small dark lymphocytes and number of epithelial reticular cells. Medulla consisted of large lymphocytes, epithelial reticular cells and Hassals corpuscles. The results showed that serum IL-2 and IL- 6 concentrations were significantly increased in vaccinated rabbits, when compared to control non-vaccinated rabbits. On contrast, the results showed that there was non-significant increase in serum TNF- $\alpha$ concentration in vaccinated rabbits when compared to control non-vaccinated rabbits. There were positive immunostaining reactions to CD4 and CD8 markers in vaccinated rabbits when compared to non-vaccinated groups, also there were positive immunostaining reaction to CD20 and CD68 markers. The present results indicated that vaccination of rabbits with VHSV increase level of cellular immune response and level of cytokines produced by the thymus.

Keywords: Rabbit, Thymus, VHSV, Cytokines, Immunohistochemistry.

(http://www.bvmj.bu.edu.eg) (BVMJ-36(1): 203-208, 2019)

\section{INTRODUCTION}

The breeding of domestic rabbits was used for multipurpose, meat and fur production and lab animals for laboratory diagnosis and research. Rabbit's meats showed high values for human consumption, it had a higher percentage of protein than other meats. The rabbits meats were highly digestible so for this reason it was recommended for sick people (Zotte, 2002). Immunity was very important process for survival of normal healthy animal, in which the body can protect itself against any attack by any foreign antigen or microbe. The body responds to any foreign either by cellular route (cell mediated immunity) which was mainly by $\mathrm{T}$ lymphocytes or by humeral route by production of antibodies specific to antigen via B lymphocytes (Jeanne, 2007).

Haley (2003) mentioned that the thymus was histologically not differs across species. It was considered as lympho-epithelial organ in which the epithelial cells form an open framework containing predominantly $\mathrm{T}$ 
lymphocytes, smaller populations of B lymphocytes and plasma cells.

Rabbit haemorrhagic disease virus extremely lethal and highly contagious virus in both domestic and wild rabbits. It caused important economic losses in the rabbit meat and fur industry, so we must control it by program of vaccination against virus (Joana et al., 2012). The present study aimed to evaluate the cellular immune response in thymus of rabbits vaccinated with viral haemorhagic septicemia vaccine (VHSV) for this purpose; we performed biochemical assays to detect cytokines (IL-2, IL-6 and TNF $\alpha$ ) and immunohistochemical techniques for detection of CD4, CD8, CD20 and CD68 which were immunomarkers of helper $\mathrm{T}$ lymphocyte, Cytotoxic T lymphocyte, B lymphocyte and macrophage respectively.

\section{MATERIALS AND METHODS}

\subsection{Animal population and samples:}

Fifteen apparently healthy rabbits at 2-monthold were collected from farm of Faculty of Agriculture, Benha University and transported to laboratory of histology at Faculty of Veterinary Medicine, where they were divided into two groups: -

- First group (Control none vaccinated) were three rabbits while, second group (Vaccinated) 12 rabbits were Injected by $0.5 \mathrm{~cm}$ VHSV S/C for each one.

The rabbits of control group were humanly sacrificed, eviscerated and subsequently, tissue specimens from the thymus were taken, the vaccinated group three rabbits were humanly sacrificed each week till the fourth week and tissue specimens from the thymus were taken. Serum samples from pre-vaccinated and postvaccinated rabbits were collected.

\subsection{Histological methods:}

The specimens were washed in distilled water for removal of blood clots and other debris, then fixed in $10 \%$ buffered neutral formalin, dehydrated in ascending grades of ethyle alcohol, cleared in xylene and embedded in paraffin wax. $5 \mu \mathrm{m}$ thick sections were cut, placed onto glass slides, and stained with Harris's haematoxylin and eosin stain for general histology of the specimens. These methods and techniques were quoted from Bancroft and Gamble (2002). Representative fields were photographed for morphology.

\subsection{Immunohistochemical methods:}

Paraffin sections were collected on positive charged microscope slides. These sections were adjacent section from the same set of slides stained for histology. Sections were deparaffinezed in xylene, rehydrated sequentially in absolute ethanol, $95 \%$ ethanol, $70 \%$ ethanol, distilled water and rinsed in phosphate buffered saline.

Antigen retrieval was done by heating the tissue section in $10 \mathrm{mM}$ citrate buffer, $\mathrm{PH} 6.0$ for 40 minutes followed by cooling at room temperature for 20 minutes. For reducing peroxidase activity, sections were treated with $3 \%$ hydrogen peroxide in methanol for 15 minutes. To block non-specific stain, sections were incubated with $10 \%$ normal goat serum for 30 minutes at room temperature. Sections were incubated with primary antibodies for CD4, CD8, CD20 and CD68 at dilution 1:200 at room temperature for 30 minutes. Then the sections were treated with goat anti-rabbit secondary antibody for 30 minutes at room temperature. Visualization was done as outlined by Kumar and Rudbeck (2009). These antibodies were detected (CD4, CD8, CD20 and CD68).

\subsection{Biochemical analysis:}

Serum samples from pre-vaccinated and postvaccinated rabbits were collected from humanly sacrificed rabbits for measurement of cytokines (IL-2 and IL-6) and TNF $\alpha$. Serum IL-2 level was determined using Rat IL-2 ELISA (Ray Biotech, Inc Company, Cath\#: ELR-IL-2) according to the manufacturer's instruction. Serum IL-6 level was determined according to the method described by (Chan 
and Perlstein, 1987). Serum TNF- $\alpha$ level was determined according to the method described by (Beyaert and Fiers, 1998).

\subsection{Statistical analysis:}

All the data were expressed as means \pm S.E. The statistical significance was evaluated by one-way analysis of variance (ANOVA) using SPSS, 18.0 software2011 and the individual comparisons were obtained by Duncan's multiple range test (DMRT). Values were considered statistically significant when $\mathrm{p}<0.05$ (Steel and Dickey, 1997).

\section{RESULTS}

\subsection{Histology of thymus:}

The thymus consisted of lobule each one had peripheral dark cortex and central light stained medulla (Fig.1).The cortex consisted of mainly small lymphocytes which characterized by dark nucleus and less epithelial reticular cells which was large cell with vesicular light nucleus and light cytoplasm (Fig.2). Medulla was paler stained than the cortex and contained large lymphocytes which had vesicular nucleus, prominent stellate or ovoid shaped epithelial reticular cells with vesicular nucleus, but light stained than that of large lymphocytes and Hassalls corpuscles (Fig.3).

\subsection{Immunohistochemical analysis:}

The representative figures showed that there was positive immunostaining reaction to CD4 markers in vaccinated group when compared to control group which show negative reaction (Fig.4).The reaction was low in first two weeks post vaccination (Fig.5) then become high in last two weeks (Fig.6). Also, there was positive immunostaining reaction to $\mathrm{CD} 8$ markers in vaccinated group when compared to control group which was negative for immunostaining (Fig.7). The reaction was low in first two weeks post vaccination (Fig.8) then become high in last two weeks (Fig.9). The results showed that there were positive immunostaining reactions to CD20 markers in vaccinated thymus (Fig.10). The immunostaining reaction to CD68 marker also appeared positively stained (Fig.11).

\subsection{Biochemical cytokine markers:}

The obtained data in table (1) showed a nonsignificant increase in serum TNF- $\alpha$ concentration in vaccinated rabbits after $1,2,3$ and 4 weeks of vaccination, when compared to control non-vaccinated group of rabbits. On contrast, the obtained data in table (1) showed a significant increase in serum IL-6 concentrations in vaccinated rabbits after 1, 2, 3 weeks of vaccination which become highly significant in fourth week of vaccination, when compared to control non-vaccinated group of rabbits. The serum IL-2 concentrations significantly increased in fourth week of vaccination, when compared to control nonvaccinated group of rabbits.

Table1. Animal groups and serum concentration level of some cytokine.

\begin{tabular}{|c|c|c|c|}
\hline Animal Groups & $\mathrm{TNF}-\alpha(\mathrm{Ug} / \mathrm{ml})$ & II $-6(\mathrm{Ug} / \mathrm{ml})$ & II $-2(\mathrm{Ug} / \mathrm{ml})$ \\
\hline Control Normal & $45.04=7.62$ & $6.71=0.92^{b}$ & $0.65 \pm 0.12^{b}$ \\
\hline First week & $51.35=7.23$ & $7.90=0.62$ & $0.83 \pm 0.07^{b}$ \\
\hline Second week & $53.99=3.90$ & $8.43=0.27$ & $0.85=0.02$ \\
\hline Third week & $58.37 \pm 1.27$ & $8.59=1.18$ & $0.94 \pm 0.17^{b}$ \\
\hline Fourth week & $61.43 \pm 1.92$ & $9.55=0.45$ & $1.34=0.04=$ \\
\hline
\end{tabular}




\section{Plate 1}

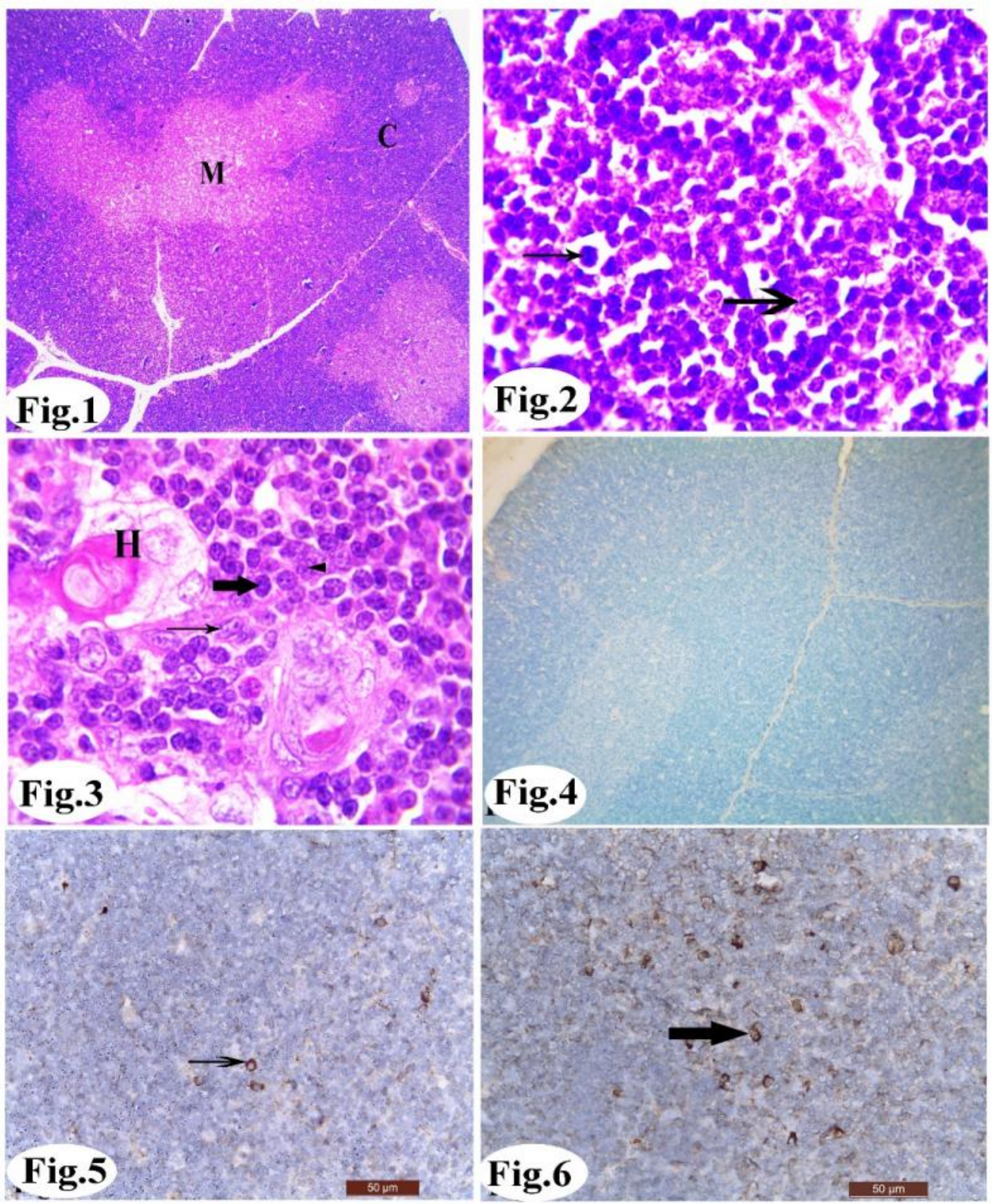

Fig.1: Photomicrograph of 2-month-old rabbits showing thymic lobule consists of peripheral dark cortex $(\mathrm{C})$ and central light stained medulla (M). H\&E X 4.

Fig.2: Photomicrograph of 2-month-old rabbits showing the cortex consists mainly of small lymphocytes (thick arrow) which characterized by dark nucleus and less epithelial reticular cells (thin arrow) which has vesicular light nucleus. H\&E X 100.

Fig.3: Photomicrograph of 2-month-old rabbits showing medulla consists of large lymphocyte (thick arrow), prominent epithelial reticular cells that may be stellate shape (thin arrow) or ovoid shape (arrowhead) and Hassalls corpuscles (H). H\&E X 100.

Fig.4: Photomicrograph of none vaccinated control rabbits showing negative immunohistochemical staining reaction for CD4. X 10.

Fig.5: Photomicrograph of post vaccinated rabbits (First two weeks) showing low positive immunohistochemical staining reaction for CD4 (arrow). X 40.

Fig.6: Photomicrograph of post vaccinated rabbits (last two weeks) showing high positive immunohistochemical staining reaction for CD4 (arrow). X 40. 
Plate 2
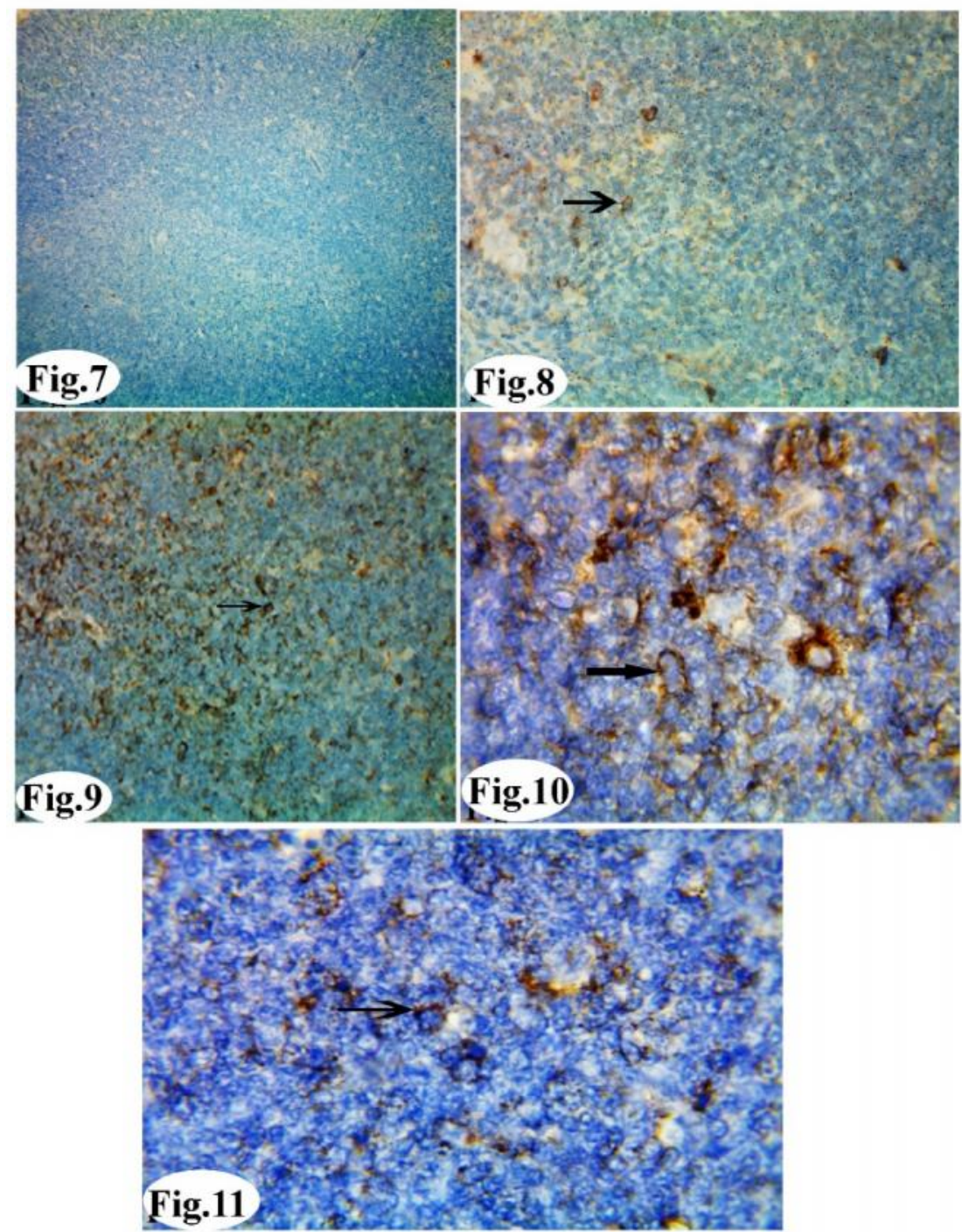

Fig.7: Photomicrograph of none vaccinated control rabbits showing negative immunohistochemical staining reaction for CD8. X 10.

Fig.8: Photomicrograph of post vaccinated rabbits (First two weeks) showing low positive immunohistochemical staining reaction for CD8 (arrow). X 40.

Fig.9: Photomicrograph of post vaccinated rabbits (last two weeks) showing high positive immunohistochemical staining reaction for CD8 (arrow). X 40.

Fig.10: Photomicrograph of post vaccinated rabbits showing positive immunohistochemical staining reaction for CD20 (arrow). X 100.

Fig.11: Photomicrograph of post vaccinated rabbits showing positive immunohistochemical staining reaction for CD68 (arrow). X 100.

\section{DISCUSSION}

The present work found that the thymus was lobulated organ consisted of peripheral dark cortex and central light stained medulla. The cortex formed from mainly small lymphocytes which characterized by dark nucleus and less epithelial reticular cells. Medulla was paler staining, less densely cellular than the cortex, and contained more large lymphocytes, prominent epithelial reticular cell and Hassalls corpuscles, this confirm the results of Kandil (1972); Haley (2003) and Pearse (2006).The level of IL-2 was significantly increased with vaccination at the fourth week which known as the T-cell growth factor. It drived the growth and differentiation of both $\mathrm{T}$ and $\mathrm{B}$ cells and induced lytic activity in natural killer cells. IL2 induced the development of $\mathrm{T}$ helper one (Th1) which its immune marker was CD4 so 
we found immune positive reaction for single positive thymocyte $\mathrm{CD} 4+$, which, in turn, induced macrophage activation, there for we found immunopositive reaction for CD68 which was the immunomarker of macrophages mentioned by Boehm et al. (1997) and Murphy and Reiner (2002). Level of IL-6 showed significant increased with vaccination as it stimulates B cells to proliferate and differentiate into plasma cells, so we found immunopositive reaction for CD 20 which was the immunomarker of B lymphocyte. Also, IL6 induces $\mathrm{CD} 4_{+} \mathrm{T}$ cells to produce greater quantities of both pro- and anti-inflammatory cytokines (Heinrich et al., 1998). Tumor necrosis factor (TNF) alpha secreted by activated monocytes and macrophages it increased but not significantly. It can activate $\mathrm{T}$ cells through its ability to induce expression of MHC class II molecules. These actions enhance antigen presentation and activate $\mathrm{T}$ cells to respond to the vaccine that triggered the initial immune response (Feldmann et al., 1996). The obtained results showed positive immuno staining reaction to CD8 in rabbits which were vaccinated as by vaccination the Tcell receptor (TCR) captures antigen and occurred activation to CD4+ $\mathrm{T}$ helper cells which differentiate to Th1, Th2, (Tsugi, 2006).The Th1 induced high-level of interferon gama (IFN- $\gamma$ ) which involved in activation of CD4 Th1 cells, CD8 cytotoxic lymphocytes and stimulate antigen presentation by MHC-I and MHC-II so increase likelihood of $\mathrm{Ag}$ capture and involvement of additional lymphocytes (Szabo et al., 2000).

\section{CONCLUSION}

Vaccination of rabbits with VHSV increase level of cellular immune response and level of cytokines produced by the thymus.

\section{REFERENCES}

Bancroft, J. D. and Gamble, M. (2002): Theory and practice of Histological Techniques. $5^{\text {th }}$ Ed., Churchill Livingstone, Medical Division of Pearson Professional Ltd.
Beyaert, R., and Fiers, W.) 1998(: Tumor necrosis factor and lympho-toxin. In Cytokines. A.R. Mire-Sluis and R. Thorpe, editors. Academic Press, San Diego, CA. 335-360.

Boehm, U.; Klamp, T.; Groot, M. and Howard, J.C. (1997): Cellular responses to interferon gamma. Ann Rev Immunol, 15:749-795.

Chan and Perlstein, Eds., (1987): Immunoassay: A Practical Guide, Academic Press: New York, p71.

Feldmann, M.; Brennan, F.M. and Maini, R.N. (1996): Role of cytokines in rheumatoid arthritis. Ann Rev Immunol, 14:397-440.

Haley, P. J. (2003): Species differences in the structure and function of the immune system. Toxicology 188:49-71.

Heinrich, P.C.; Behrmann, I.; Muller-Newen, G.; Schaper, F.; and Graeve, L. (1998): Interleukin-6-type cytokine signaling through the gp130/Jak/STAT pathway. Biochem J. 334:297-314.

Jeanne, K. (2007): Understanding the Immune System How It Work. National Institute of Allergy and Infectious Diseases (NIH). Publication No, 7.

Joana, A.; Wessel, V.; Jacques, L. and Pedro, J. (2012): Rabbit haemorrhagic disease (RHD) and rabbit haemorrhagic disease virus (RHDV): a review. Veterinary Research, 12:43.

Kandil, M.H. (1972): Histological and histochemical studies on the thymus gland of the camel. M.V.SC. thesis, Fac.Vet. Med. Cairo University.

Kumar, G., and Rudbeck, L. (2009): Education Guide: Immunohistochemical Staining Methods. 5th Edition. Dako North America, Carpenteria, California, U.S.A.

Murphy, K.M. and Reiner, S.L. (2002): The lineage decisions of helper $\mathrm{T}$ cells. Nature Rev Immunol, 2:933-944.

Pearse, G. (2006): Normal Structure, Function and Histology of the Thymus. Toxicologic Pathology, 34:504-514.

Steel, R.; Torrie, J. and Dickey, D. (1997): Principles and procedures of Statistics: A Biometrical Approach, 3rd ed., McGraw-Hill, New York, NY.

Szabo, S.J.; Costa, G.L; Zhang, X. and Glimcher, L.H (2000): A novel transcription factor, T-bet, directs Th1 lineage commitment. Cell, 100:655-669.

Tsugi, N.M. (2006): Antigen-specific CD4+T cells in the intestine. Inflamm Aller Drug Targets, 5:191-201.

Zotte,A.D. (2002): Perception of rabbit meat quality and major factors influencing the rabbit carcass and meat quality. Livestock production science. 75:11-32. 